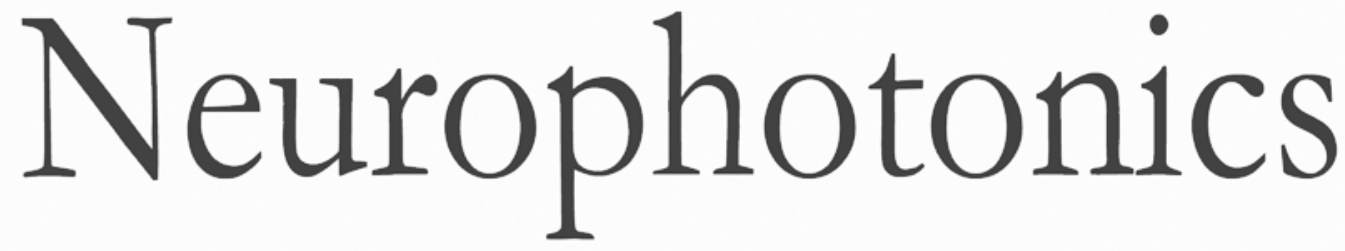

\title{
Handheld spectrally encoded coherence tomography and reflectometry for motion-corrected ophthalmic optical coherence tomography and optical coherence tomography angiography
}

Joseph D. Malone

Mohamed T. El-Haddad

Suhaas S. Yerramreddy

Ipek Oguz

Yuankai K. Tao 


\title{
Handheld spectrally encoded coherence tomography and reflectometry for motion-corrected ophthalmic optical coherence tomography and optical coherence tomography angiography
}

\author{
Joseph D. Malone, ${ }^{a}$ Mohamed T. El-Haddad, ${ }^{a}$ Suhaas S. Yerramreddy, ${ }^{b}$ Ipek Oguz, ${ }^{b}$ and Yuankai K. Tao ${ }^{a, *}$ \\ ${ }^{a}$ Vanderbilt University, Department of Biomedical Engineering, Nashville, Tennessee, United States \\ bVanderbilt University, Department of Electrical Engineering and Computer Science, Nashville, Tennessee, United States
}

\begin{abstract}
Optical coherence tomography (OCT) is the gold standard for quantitative ophthalmic imaging. The majority of commercial and research systems require patients to fixate and be imaged in a seated upright position, which limits the ability to perform ophthalmic imaging in bedridden or pediatric patients. Handheld OCT devices overcome this limitation, but image quality often suffers due to a lack of real-time aiming and patient eye and photographer motion. We describe a handheld spectrally encoded coherence tomography and reflectometry (SECTR) system that enables simultaneous en face reflectance and cross-sectional OCT imaging. The handheld probe utilizes a custom double-pass scan lens for fully telecentric OCT scanning with a compact optomechanical design and a rapid-prototyped enclosure to reduce the overall system size and weight. We also introduce a variable velocity scan waveform that allows for simultaneous acquisition of densely sampled OCT angiography (OCTA) volumes and widefield reflectance images, which enables highresolution vascular imaging with precision motion-tracking for volumetric motion correction and multivolumetric mosaicking. Finally, we demonstrate in vivo human retinal OCT and OCT angiography (OCTA) imaging using handheld SECTR on a healthy volunteer. Clinical translation of handheld SECTR will allow for high-speed, motion-corrected widefield OCT and OCTA imaging in bedridden and pediatric patients who may benefit ophthalmic disease diagnosis and monitoring. () The Authors. Published by SPIE under a Creative Commons Attribution 4.0 Unported License. Distribution or reproduction of this work in whole or in part requires full attribution of the original publication, including its DOI. [DOI: 10.1117/1 .NPh.6.4.041102]

Keywords: multimodal imaging; optical coherence tomography; angiography; handheld imaging; motion correction.

Paper 19034SSR received Apr. 8, 2019; accepted for publication Jun. 12, 2019; published online Jul. 3, 2019.
\end{abstract}

\section{Introduction}

Optical coherence tomography (OCT) is the gold standard for quantitative ophthalmic imaging and enables volumetric visualization of subsurface tissue microstructures. ${ }^{1}$ The majority of current-generation commercial and research OCT systems utilize a benchtop design that requires patients to be imaged in a seated upright position. However, visualization of peripheral retinal structures is challenging because of the limited mechanical range of motion of the OCT scan head. Moreover, long acquisition protocols, such as those used for OCT angiography (OCTA), require extended patient fixation. All of these aforementioned limitations make OCT/OCTA imaging in uncooperative, bedridden, or pediatric patients exceedingly difficult using conventional benchtop systems. Although several handheld OCT probes have been developed specifically to image supine patients, these systems have not adequately addressed significant functional limitations of point-of-care ophthalmic imaging, such as motion artifacts and the need for real-time aiming and retinal tracking. ${ }^{2-6}$

OCT motion artifacts are dominated by microsaccades (15 to $150 \mathrm{deg} / \mathrm{s})$ and ocular drift $(0.5 \mathrm{deg} / \mathrm{s}) .^{7-9}$ In handheld imaging, these artifacts are further compounded by photographer motion

*Address all correspondence to Yuankai K. Tao, E-mail: yuankai.tao@ vanderbilt.edu and tremor, which is on the order of 8 to $12 \mathrm{~Hz} .^{2,10,11}$ These artifacts can affect the anatomic accuracy of OCT volumetric data and lead to errors in quantitative image analysis. Motion also significantly affects OCTA postprocessing, resulting in regions of high decorrelation contrast and vascular discontinuities. $^{12}$ Previously described methods for reducing OCT motion artifacts include higher speed image acquisition, ${ }^{13-15}$ combinations of scan patterns with computational postprocessing, ${ }^{5,16-19}$ and optical tracking. ${ }^{20-22}$ However, the added system and/or computational complexity often limit their application to benchtop systems. Increasing OCT frame rates reduces motion artifacts, but there are inherent trade-offs between imaging speed, signal-to-noise ratio, sampling density, and field of view (FOV). ${ }^{23,24}$ In addition, computational motion-artifact correction has been implemented using orthogonally scanned OCT volumes, but reconstruction accuracy is dependent on the total number of repeated volumes and overlapping fiducials, making it unfavorable in time-restricted clinical imaging. ${ }^{16}$

Multimodal technologies have been developed for real-time aiming and high-speed retinal tracking, which combine OCT with en face reflectance imaging using video ophthalmoscopy or scanning laser ophthalmoscopy (SLO). However, these multimodal systems are complex and often include dedicated illumination, imaging, and detection optics and have proven difficult to translate clinically. ${ }^{20,22,25,26}$ Both handheld OCT and multimodal ophthalmic imaging probes have been demonstrated using 
resonant or MEMS scanners to reduce size and increase scan speeds; however, these can have limited FOVs, nonlinear scan trajectories that require additional postprocessing, and different frame rates for each modality, thereby lacking temporal coregistration. ${ }^{27,28}$ We address the aforementioned limitations of existing multimodal handheld ophthalmic probes by using spectrally encoded coherence tomography and reflectometry (SECTR), which integrates OCT with spectrally encoded reflectance (SER) imaging. ${ }^{29-31}$ While conventional SLO uses pointscan illumination and confocal detection, SER uses line-scan illumination by spectrally dispersing a broadband light source and encoding spatial position as a function of wavelength. SER and OCT optical paths are coaligned and signals are simultaneously sampled using a shared high-speed digitizer for reduced system complexity and simultaneous acquisition of en face SER frames with each OCT cross section at $>200$ frames per second. Finally, SECTR uses a shared galvanometer scanner that ensures inherent spatiotemporal coregistration between OCT and SER data.

Here, we demonstrate SER-based retinal tracking for motion correction in OCT volumes using a handheld SECTR imaging probe. In vivo human retinal imaging was performed in a healthy volunteer and motion artifacts in OCT/OCTA data were compared with and without correction. We also developed a custom scan pattern that densely samples a region of interest (ROI) within a sparsely sampled SECTR volume to enable OCTA imaging of retinal microvasculature while providing widefield retinal fiducials for motion tracking. Previous studies have demonstrated the effectiveness of handheld OCT for monitoring retinopathy of prematurity in infants, ${ }^{10,32-36}$ evaluating structural changes in uncooperative Parkinson's patients, ${ }^{37}$ and visualizing abnormal retinal structures. ${ }^{38-40}$ However, these studies were performed using OCT systems that had limited aiming and retinal-tracking functionality. Similarly, while groups recently published handheld OCT probes optimized for pediatric imaging, ${ }^{41,42}$ these devices lack integrated motion tracking, widefield imaging, and real-time retinal-aiming capabilities that are necessary to overcome current limitations in point-of-care ophthalmic diagnostic imaging. We believe that our handheld SECTR imaging probe will improve image quality, enable robust structural and functional imaging, and benefit clinical ergonomics for imaging bedridden and pediatric patients.

\section{Methods}

\subsection{Spectrally Encoded Coherence Tomography and Reflectometry Engine and Data Acquisition}

SECTR imaging was performed using a previously described OCT engine with a $200 \mathrm{kHz}, 1060$-nm center wavelength sweptsource laser (Axsun) optically buffered to $400 \mathrm{kHz}$. $^{31}$ All system specifications and components were identical except for the use of a transmissive reference arm for improved power throughput [Fig. 1(a)]. The OCT and SER sample fibers from the engine [Fig. 1(a), red and blue boxes, respectively] are coupled to the corresponding collimating optics in the handheld probe [Fig. 1(b), $f_{c}$ and PM, respectively].

\subsection{Double-Pass Scan Lens}

Galvanometer scanning modules conventionally use closely positioned $x$-axis and $y$-axis scanner pairs to reduce system size and complexity. In this configuration, neither scanner is conjugated to the pupil plane, which results in a nontelecentric focal plane. Telecentric scanning relays can be implemented, but require an additional telescope to optically relay conjugate pupil planes to separated scanning mirrors. We introduce a double-pass scan lens (DPSL) that enables dual-axis telecentric scanning using a single-lens configuration [Figs. 1(b) and 1(c)]. (a)

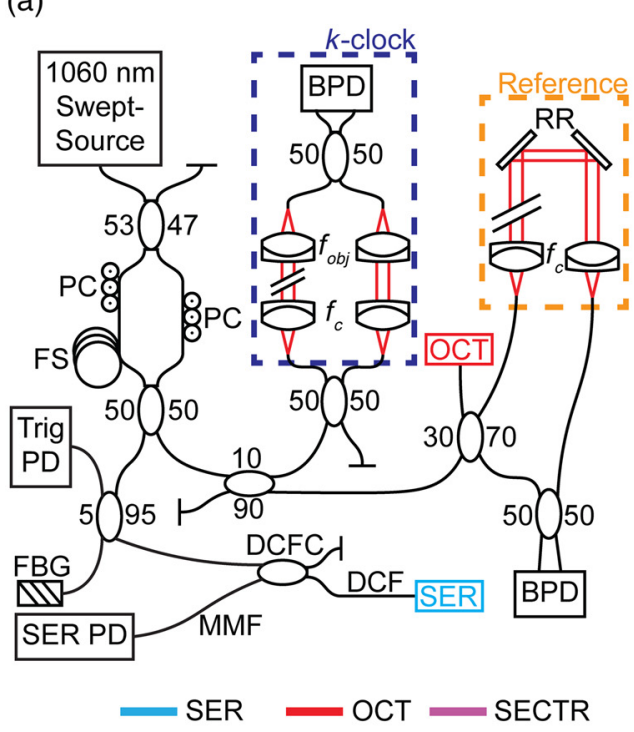

(b)

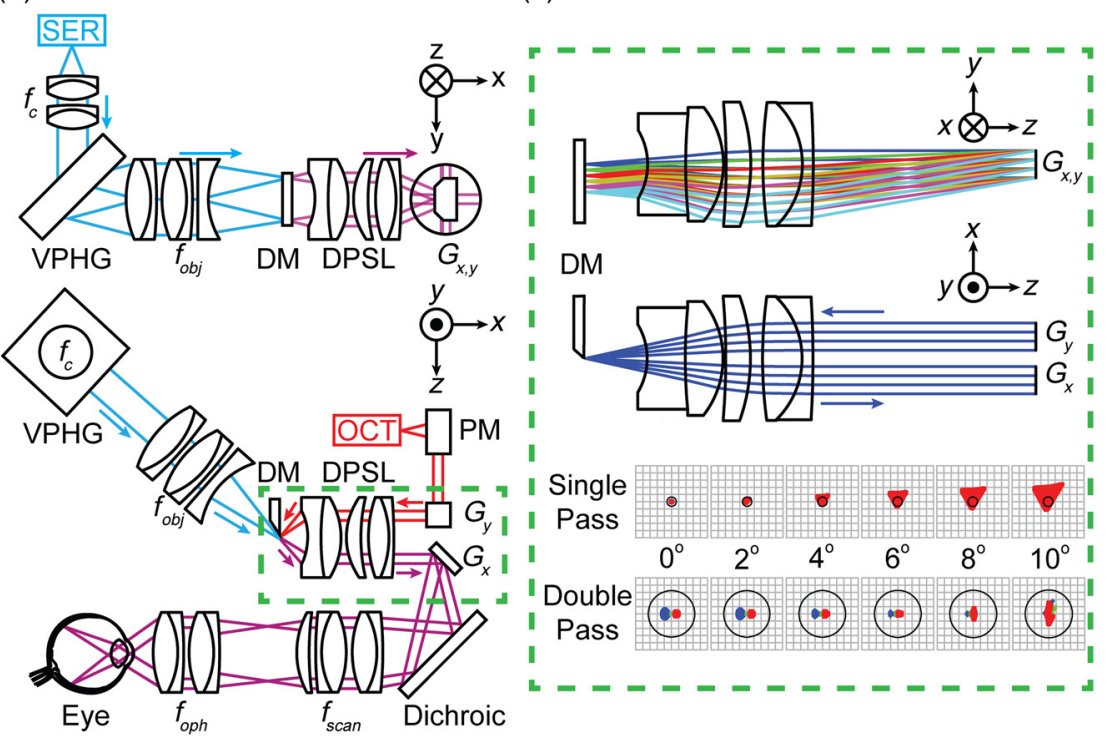

Fig. 1 Optical design and schematic representation: (a) SECTR engine and (b) handheld optical system schematics. (c) Orthogonal views and simulated single- and double-pass spot diagrams through the DPSL (Airy radius $=12.91 \mu \mathrm{m}$ ). Arrows show direction of light propagation. PC, polarization controller; PD, photodiode; FBG, fiber Bragg grating; BPD, balanced photodetector; DCFC, double-clad fiber coupler; MMF, multimode fiber; DCF, double-clad fiber; RR, retroreflector; VPHG, volume-phase holographic grating; DM, d-shaped pickoff mirror; PM, parabolic mirror; $G_{x, y}$, galvanometer; $f_{c}$, collimating lens; $f_{\mathrm{obj}}$, focusing lens; DPSL, double-pass scan lens; $f_{\mathrm{scan}}$, scan lens; and $f_{\mathrm{oph}}$, ophthalmic lens. 
The DPSL was custom-designed using CodeV (Synopsys, Inc.) to optimize performance over a \pm 10 - deg optical FOV. Figure 1(c) shows representative spot diagrams for single-pass performance at the mirror plane (DM) and double-pass performance at the fast-axis galvanometer $\left(G_{x}\right)$ for $0 \mathrm{deg}$ to $10 \mathrm{deg}$. The simulated spots in the mirror plane [Fig. 1(c)] show significant single-pass aberrations dominated by coma, but the lens corrects for this upon a second pass, resulting in diffractionlimited performance across the entire FOV. The double-pass spot sizes were simulated using a paraxial imaging lens with a focal length of $30 \mathrm{~mm}$ and an Airy radius of $12.91 \mu \mathrm{m}$ to avoid confounding aberrations not inherent to the DPSL and allow for direct comparison between single- and double-pass performances.

\subsection{Optical Design}

SECTR imaging performance was simulated and optimized using ZEMAX (ZEMAX, LLC) with stock and custom optical components [Fig. 1(b)]. In the OCT path, a single-mode fiber $(\mathrm{NA}=0.14)$ was collimated using a 90-deg off-axis parabolic mirror [Fig. 1(b), PM, Thorlabs, RC04FC-PO1] to a 4-mm diameter. The beam was then scanned using the slow-axis galvanometer $\left(G_{y}\right.$, ScannerMax, Pangolin Laser Systems, Inc.) positioned at a 4-mm offset from the optical axis and focused telecentrically onto a D-shaped pickoff mirror (DM) using the DPSL [Fig. 1(c)]. The beam was reflected and backpropagated through the DPSL to the fast-axis galvanometer $\left(G_{x}\right)$. The galvanometers were separated by $8-\mathrm{mm}$ center to center to accommodate the physical size of each mirror. SER used a double-clad fiber (DCF) for coaxial single-mode illumination and multimode collection that was oriented at $29 \mathrm{deg}$ with respect to the optical axis to compensate for a custom DCF termination (Sec. 2.4). Single-mode SER illumination was collimated to
$10 \mathrm{~mm}$ using two achromatic doublets with a combined focal length of $26.2 \mathrm{~mm}$ [Fig. 1(b), $f_{c}$ ], and spectrally dispersed using a 1379 lines/mm polarization-independent transmission grating (PING-Sample-420, Ibsen Photonics, A/S). The dispersed beam was then focused to an 11.5-mm line using a 54-mm focal length lens $\left(f_{\text {obj }}\right)$ and combined with the OCT path across the D-shaped mirror (DM). SER and OCT propagated collinearly with a 50- $\mu \mathrm{m}$ separation through the DPSL, a shared galvanometer $\left(G_{x}\right)$, a dichroic hot mirror (dichroic, 43-843; Edmund Optics, Inc.), and a $2 \times$ demagnifying telescope $\left(f_{\text {scan }}\right.$ and $\left.f_{\text {oph }}\right)$ to a 2-mm diameter spot on the pupil. The dichroic mirror was added to allow for the placement of an optional visible-light fixation target.

A modified version of the Polans eye model was used to accurately model aberrations through the human eye. ${ }^{43,44}$ Figure 2 shows simulated spot diagrams for OCT and SER over $\mathrm{a} \pm 20$ - deg FOV on the retina. The Airy radii for OCT and SER were 9.8 and $9.6 \mu \mathrm{m}$, respectively. We achieved diffractionlimited OCT performance over the central $13 \mathrm{deg}$ and up to twice the diffraction limit at the FOV periphery (RMS spot size). OCT spot sizes at the peripheral FOV were limited by the use of $\varnothing 1$ " optics, which was necessary to maintain a compact form factor for handheld imaging. SER spots were twice the diffraction limit on axis and up to six times the diffraction limit at the periphery. SER imaging performance was mainly degraded by single-pass propagation through the DPSL, which induced significant coma and astigmatism across the field. SER is primarily used for retinal aiming during image acquisition and motion tracking in postprocessing and, thus, SECTR performance was not significantly impacted by SER lateral resolution. It is possible to precompensate for these aberrations using customdesigned collimator and objective lenses [Fig. 1(b), $f_{c}$ and $f_{\text {obj }}$. However, the achievable SER resolution with our current system design was more than sufficient for retinal aiming and

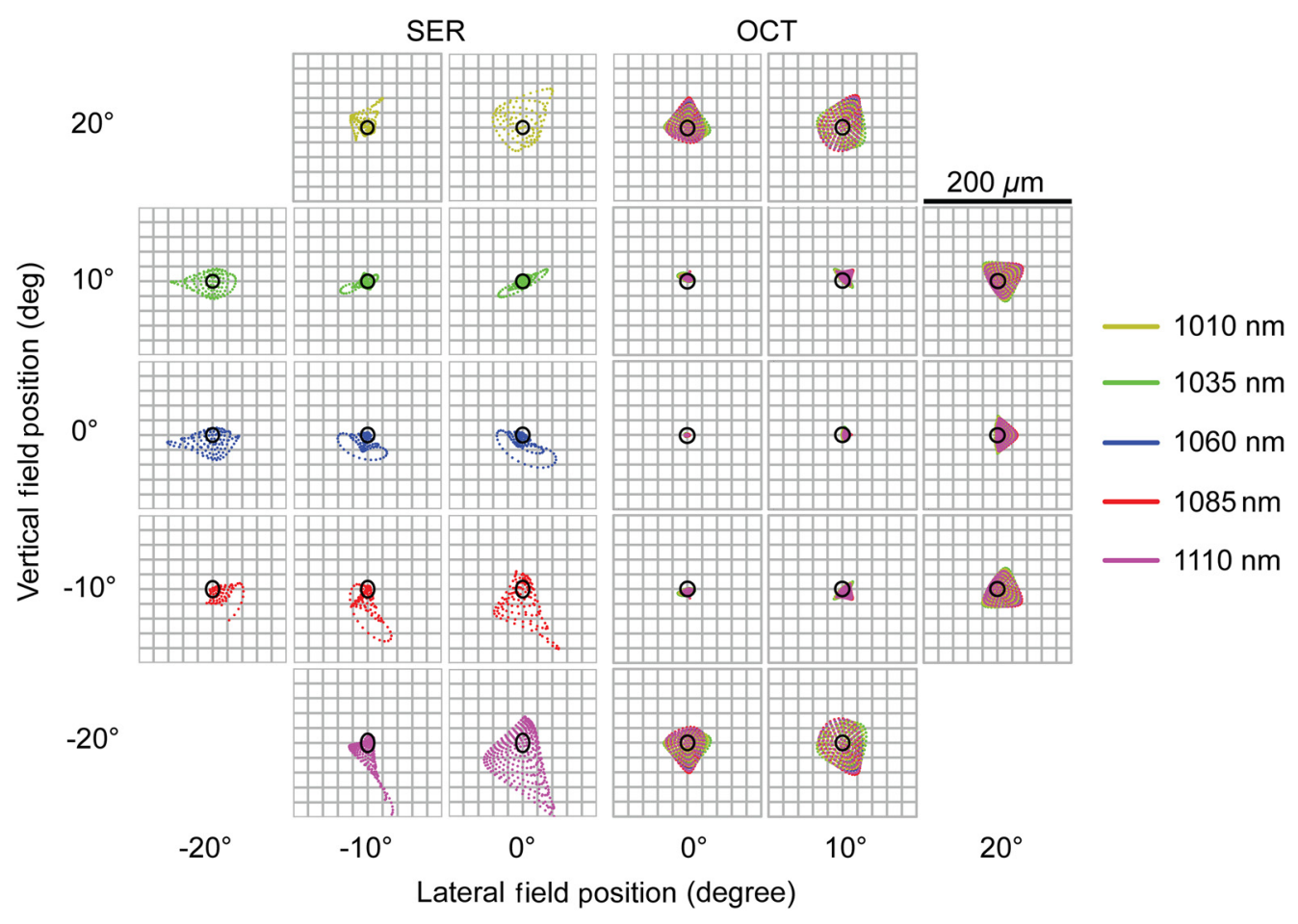

Fig. 2 Spot diagrams in retinal plane of the Polans eye model. OCT and SER spots were simulated across a \pm 20 - deg FOV. Airy radius: $9.8 \mu \mathrm{m}$ (OCT) and $9.6 \mu \mathrm{m}$ (SER). 
motion tracking and, thus, stock collimator and objective lenses were used to reduce overall system cost and complexity.

\subsection{Double-Clad Fiber Coupler End-Face Reflections}

Single-mode illumination and multimode collection using a DCF improves SER collection efficiency while maintaining lateral resolution. Multimode collection also provides speckle contrast reduction through incoherent averaging of multiple spatial modes of backscattered light on the detector. Unfortunately, DCF end-face reflections preferentially couple into the inner cladding and saturate backscattered SER signals from the retina [Fig. 3(a)]. We previously developed a method to reduce these end-face reflections by index-matching the DCF end-face to an angled wedge prism, which spatially offset reflections from the glass-to-air interface away from the inner cladding of the DCF [Fig. 3(b)] ${ }^{31}$ Although this achieved a $12.5 \times$ reduction in backcoupled end-face reflection intensity, the wedge prism was bulky and required repeated application of index-matching gel.

Here, we describe an improved method for removing DCF end-face reflections using an angle-polished no-core fiber (NCF) termination. Optimal NCF length and polish angle [Fig. 3(e)] were simulated in MATLAB (MathWorks, Inc.) and ZEMAX using DCF manufacturer specifications for fiber numerical aperture (NA), diameters, and refractive index (SMM900, Fibercore). Simulations ensured that the NCF end-face $(125-\mu \mathrm{m}$ diameter) did not clip the SER illumination beam and reflections were completely spatially offset from the inner cladding [Fig. 3(d)]. The plot in Fig. 3(e) shows red and blue regions corresponding to suitable output beam diameters and minimum fiber polish angles to ensure no backcoupling into the inner cladding or beam clipping at the NCF output, respectively. Furthermore, the dotted black line shows a maximum beam diameter of $125 \mu \mathrm{m}$ (the physical diameter of the NCF), which limits the maximum fiber length to $0.35 \mathrm{~mm}$. The gray region, where the blue and red regions overlap, is all acceptable combinations of fiber polish angle and NCF length for a maximum beam diameter of $125 \mu \mathrm{m}$. As a result of these simulations, a $210-\mu \mathrm{m}$ length of NCF was polished to 20 deg to optimally terminate a DCF with single-mode core NA of 0.19 , inner cladding NA of 0.26 , and inner cladding diameter of $125 \mu \mathrm{m}$. Leakage between the single-mode illumination and inner cladding was observed, so NCF termination length and angle were optimized assuming 0.26-NA illumination output at the DCF face. A short length of NCF (FG125LA, Thorlabs, Inc.) was first fusion-spliced to a flatcleaved DCF and then cleaved, leaving $250 \mu \mathrm{m}$ of NCF past the splice-joint. The fiber was fed through an FC/PC connector and epoxied under microscopy guidance such that $40 \mu \mathrm{m}$ of NCF protruded from the ferrule tip. The fiber and ferrule were then polished to $20 \mathrm{deg}$. Polishing both the fiber and ceramic ferrule enabled routine inspection and cleaning of the fiber face without damaging the NCF termination.

Three illumination configurations were used to characterize the backreflection intensity of our DCF termination: DCF in air, DCF with index-matching gel, and DCF with NCF termination [Figs. 3(a), 3(c), and 3(d), respectively]. Backreflection intensity from the DCF with index-matching gel was used as a reflectionfree baseline. The measurements were performed with no downstream optics to decouple end-face from other system reflections. The intensity of the backreflected light incident on the SER photodiode was recorded over several sweeps of the laser for each configuration [Fig. 3(f)]. All measurements were reported relative to the backreflection from the DCF in air. We measured 25-dB average backreflection reduction using our NCF termination, which was comparable to the index-matching

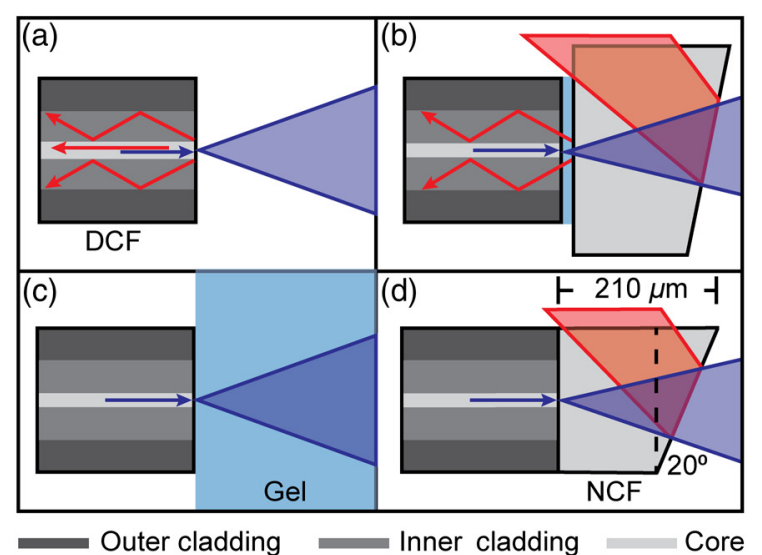

(e)

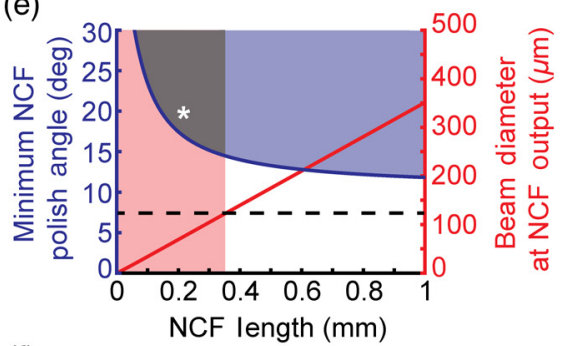

(f)

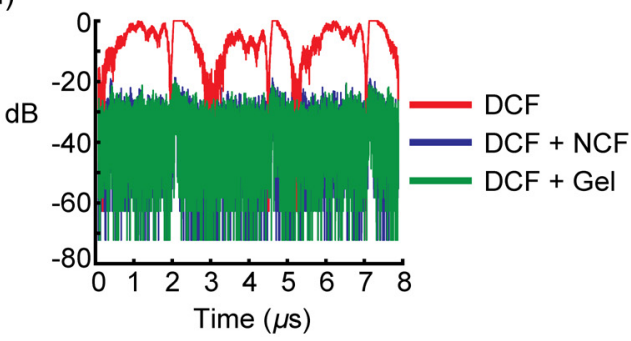

Fig. 3 DCF end-face termination. (a) Flat-polished DCF with blue and red rays representing illumination and backreflection, respectively. (b) Previously described backreflection reduction configuration using a wedge prism coupled to the DCF with index-matching gel. DCF illumination with (c) index-matching gel and (d) NCF termination. (e) MATLAB simulation for optimal polish angle and fiber length of NCF termination. The blue and red shaded regions represent acceptable polish angles and beam diameters to ensure no backcoupling or beam clipping, respectively. The black dotted line denotes the maximum beam diameter cut-off for a fiber diameter of $125 \mu \mathrm{m}$. The gray region shows the overlap between suitable NCF lengths and polish angles and the white asterisk shows the fabrication parameters of our NCF termination. (f) Backreflection measurements for fiber configurations (a), (c), and (d). 
gel baseline and a significant improvement over our previously reported wedge prism termination. ${ }^{31}$

\subsection{Optomechanical Design and Housing}

A combination of stock and custom optomechanical components was integrated into a high-resolution rapid-prototyped enclosure to achieve a compact, lightweight, and robust handheld SECTR probe (Fig. 4). The optimized optical design was exported from ZEMAX to SolidWorks (Dassault Systèmes, SE) for mechanical design and optomechanical components were imported from available manufacturer models. Fold mirrors were used to reduce the size of the handheld, and a custom galvanometer holder was designed to reduce bulk while maintaining precise $x-y$ galvanometer separation and alignment. Optical elements were directly mounted onto the rapid prototyped enclosure with extruded cantilever-style snap-fit mounts and pedestals to reduce overall system bulk and weight. The ophthalmic lens [Fig. 1(b), $f_{\text {oph }}$ ] was mounted in a zoom housing to compensate for optical power differences between patient eyes. When completely assembled, the SECTR probe measured $14 \times 6.8 \times 10 \mathrm{~cm}$ (length $\times$ width $\times$ height $)$ and weighed $752 \mathrm{~g}$.

\subsection{Motion-Correction and Multivolumetric Mosaicking}

We previously described methods for multivolumetric registration and mosaicking of SECTR volumes. ${ }^{31}$ In this work, we developed a modified algorithm to correct for motion artifacts and mosaic OCTA volumes. Lateral (i.e., horizontal and vertical) shifts were computed from serial SER images and axial shifts were computed from OCT B-scans using discrete Fourier transform registration. ${ }^{45}$ The measured lateral and axial shifts were then applied to each corresponding B-scan, and OCTA was performed using singular value decomposition on each motion- corrected volume. ${ }^{46,47}$ SER shifts were scaled to corresponding OCT dimensions by first calculating the ratio between the SER and OCT FOVs and then empirically minimizing the vessel discontinuities. The scaling ratio is constant for a given set of scan parameters and, thus, manual scaling was only performed once for each set of structural and vascular data acquired in this study. OCTA projections were then roughly aligned manually using overlapping fiducials. The Frangi filter was applied to each OCTA projection to highlight vascular features. ${ }^{48}$ The "vesselness" feature maps were then automatically aligned using an intensity-based deformable registration method and the resulting transformations were applied to the original OCTA volumes. Finally, the aligned OCTA volumes were blended together to obtain a final mosaic of the overlapping datasets.

\subsection{Variable Velocity Waveform Imaging}

Lateral motion estimation depends on the contrast and the number of retinal fiducials present in SER frames (e.g., blood vessels or optic nerve head). Thus, the lack of fiducials in the peripheral retina and fovea limits the accuracy and robustness of multivolumetric registration and mosaicking in these regions. These limitations are further exacerbated in OCTA, which requires small densely sampled volumes to achieve high vascular resolution. SECTR is uniquely suited for retinal OCTA mosaicking because widefield SER images may be acquired concurrently with small densely sampled OCTA volumes to ensure that motion-tracking fiducials are consistently visible. We achieved this using a custom-designed scan waveform to drive the shared SER and OCT fast-axis galvanometer such that the scan velocity was slow within the ROI to ensure optimal OCTA sampling density and high outside of the ROI [Fig. 5(a)]. Applying this scan waveform allowed us to acquire widefield SER frames for motion tracking and a critically sampled OCT volume for

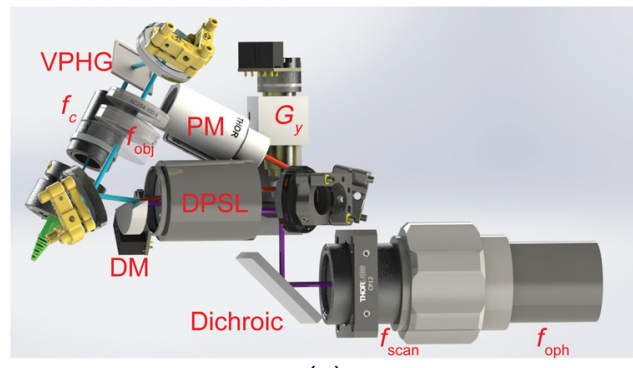

(a)

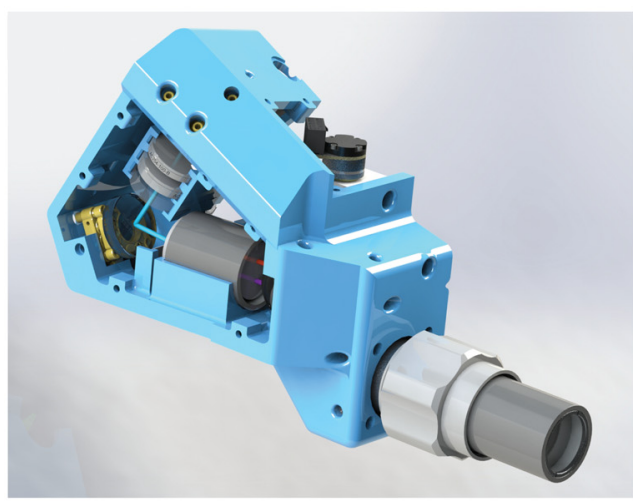

(b)

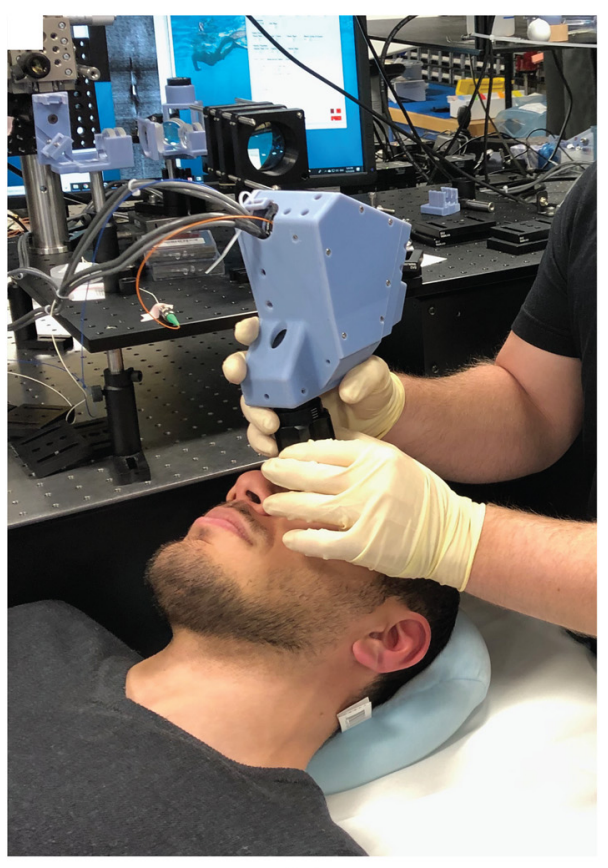

(c)

Fig. 4 Handheld SECTR probe. CAD renderings of the (a) optical and optomechanical layout and (b) rapid-prototyped enclosure, and (c) picture of SECTR probe being used to image a healthy volunteer. 
(a)

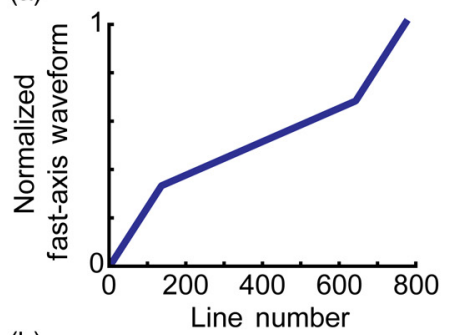

(b)

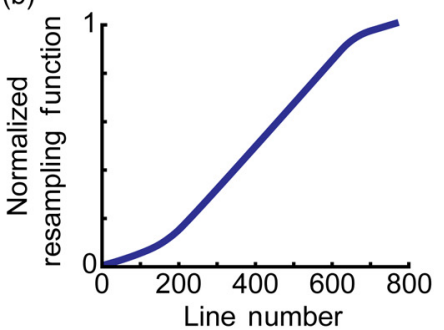

(c)

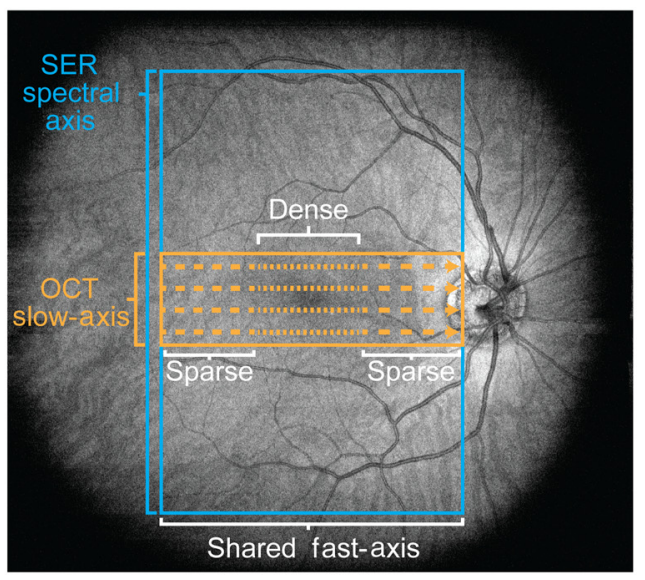

Fig. 5 Variable velocity scan waveform imaging. (a) Variable velocity scan waveform has a normalized slope of two between lines 0 to 124 and 625 to 750 (fast) and 0.5 between lines 125 to 624 (slow). (b) Corresponding linear resampling function. (c) Schematic representation of the SECTR FOV overlaid on a widefield OCT projection. Dotted orange lines represent the shared fast-axis scan trajectory and sampling density. Blue and orange boxes show the SER and OCT FOVs, respectively.

angiography at a fraction of the total acquisition time of a conventional critically sampled volume across the same FOV. In postprocessing, the SER and OCT data were resampled to linearize the line spacing and to correct the image distortions resulting from the scan waveform [Fig. 5(b)]. The resampling function was obtained by first imaging a grid chart with a line spacing of $100 \mu \mathrm{m}$. The signal peak positions corresponding to the grid lines were then extracted from OCT frames and linearized. Smooth transitions between the fast and slow scan velocity regions in the resampling function was a result of low-pass filtering of the scan waveform by the galvanometer controller.

\section{Results}

\subsection{Optical Performance}

Handheld SECTR optical performance was evaluated by imaging a USAF-1951 resolution chart. All resolution tests were performed in the intermediate image plane before the ophthalmic relay lens [Fig. 1(b), $f_{\text {oph }}$ ]. Figures 6(a) and 6(b) show resolution chart images for SER and OCT sampled at $2560 \times$ $2000 \times 2000$ pixels $($ spectral $\times$ lateral $\times$ frames $)$ over a $1.1-\mathrm{mm}^{2}$ region. Images were oversampled to ensure that the resolution was limited by optical performance rather than sampling artifacts. OCT fast- and slow-axes resolved group 6 elements 4 and $2(90.5$ and $71.8 \mathrm{lp} / \mathrm{mm})$, respectively, and SER resolved group 5 element 1 (32 lp/mm) [Figs. 6(a) and 6(b)].

\subsection{In Vivo Widefield Human Imaging}

Human retinal imaging was performed under an internal review board approved protocol. A healthy volunteer was imaged supine to simulate clinical imaging with an untrained photographer. The SER and OCT power incident on the pupil were $3.6 \mathrm{~mW}$ (extended line illumination) and $1.2 \mathrm{~mW}$ (point

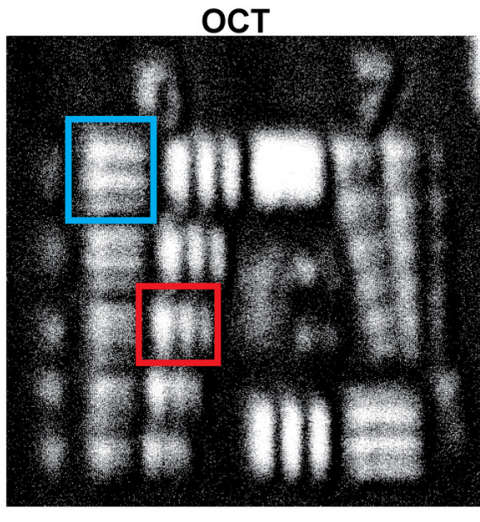

(a)

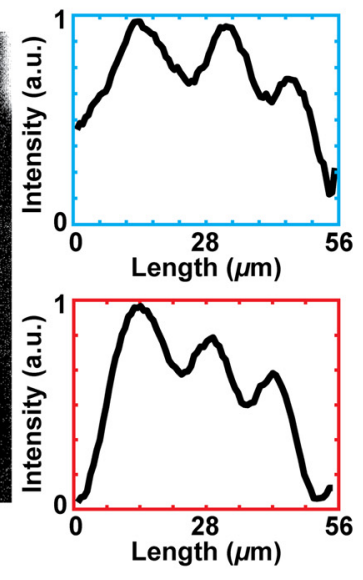

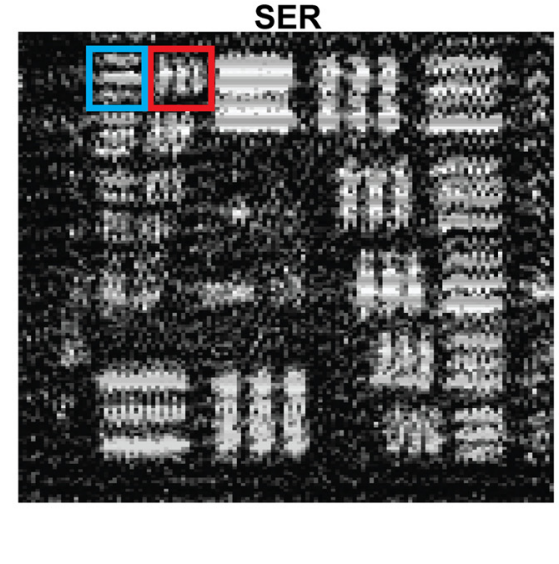

(b)

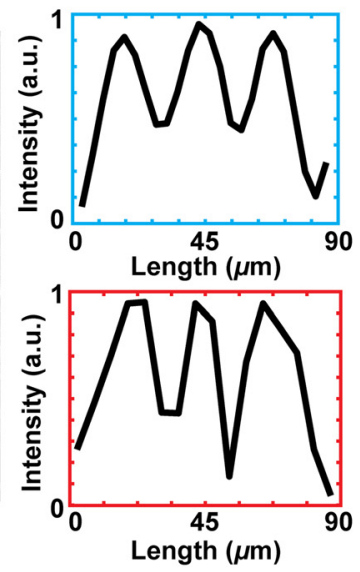

Fig. 6 Validation of handheld SECTR imaging performance. (a) En face projection of USAF-1951 resolution chart imaged with the OCT. Red and blue plots show intensity cross sections of group 6 elements 4 and 2, respectively. (b) SER image with cross sections of group 5 element 1. 


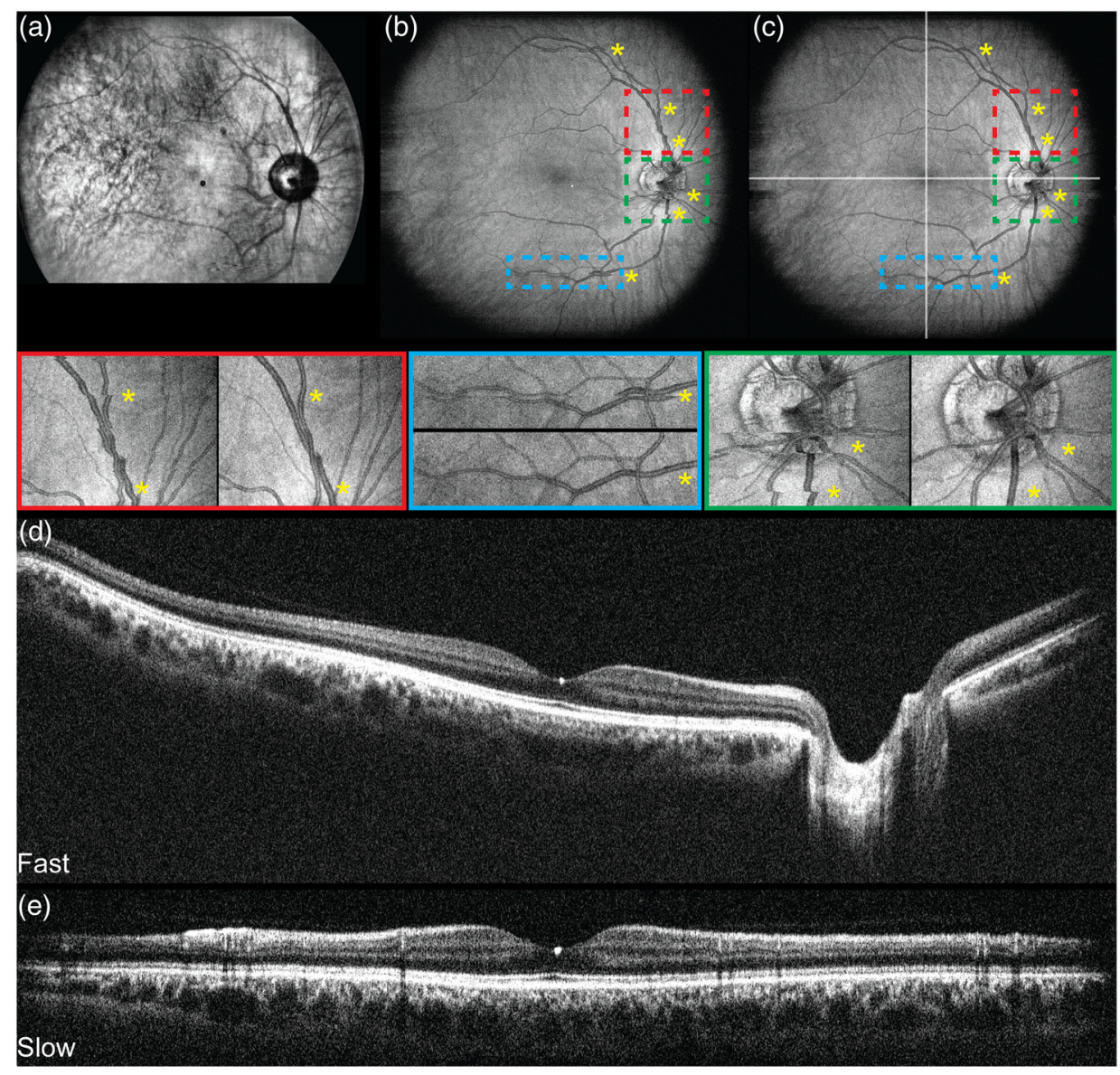

Fig. 7 In vivo human retinal imaging performed on a healthy volunteer. (a) Four-frame average SER and average intensity en face projections of (b) raw and (c) motion-corrected OCT volumes. Red, blue, and green boxes show magnified examples of photographer tremor, slow-axis motion, and fast-axis motion (asterisks) before and after motion correction. Representative (d) fast-axis and (e) slow-axis OCT cross sections from the motion-corrected volume (white lines).

illumination), respectively, and well below the ANSI maximum permissible exposure limit for in vivo human retinal imaging (ANSI Z80-36306). Widefield images were sampled at $2560 \times$ $1500 \times 1500$ pixel $($ spectral $\times$ lateral $\times$ frames) per volume in 6.125 s. Datasets were oversampled to demonstrate probe stability and motion-correction postprocessing methods over an extended acquisition time. Figure 7 shows a four-frame averaged SER frame and raw and motion-corrected OCT en face projections [Figs. 7(a)-7(c)].

\subsection{Angiography with Variable Velocity Scan Waveform}

Motion-corrected OCTA was performed using variable velocity scan waveform imaging (Secs. 2.6 and 2.7) with the handheld SECTR probe in a stabilized mount. Figures 8(a) and 8(b) show representative SER frames before and after resampling, respectively, and Figs. 8(c) and 8(d) show corresponding OCTA projections. The volume was sampled with $2560 \times 750 \times$ 500 pixel (spectral $\times$ lines $\times$ frames) with four repeated frames. Total imaging time was $5.25 \mathrm{~s}$. No obvious difference in resolution or contrast was observed on SER between the fast and slow scan regions, whereas OCTA projections showed significant vessel enhancement in the central densely sampled foveal region.

\subsection{Widefield Multivolumetric Mosaicking}

Handheld SECTR OCTA was performed on a second supine healthy volunteer by a second untrained photographer. Multivolumetric mosaicking was performed on three overlapping volumes from the fovea out to the optic nerve. Volumes were sampled with $2560 \times 500 \times 400$ pixel (spectral $\times$ lines $\times$ frames) and four repeated frames in $3.2 \mathrm{~s}$. Figures 9(a)-9(c) show each motion-corrected volume. Although the volumes were acquired over the same FOV and sampling density, we can see variations in volume height due to compression and/or stretching of the data following motion correction. This effect results from motion in the OCT slow axis and is clearly visible in Fig. 9(a) where vertical motion resulted in missing data (black horizontal lines). Edges of individual volumes [Figs. 9(a)-9(c)] were cropped prior to mosaicking to remove distorted regions resulting from nonlinear scan trajectories during galvanometer flyback. Mosaicking was performed using the methods described in Sec. 2.6 [Fig. 9(d)] and demonstrates the feasibility of widefield handheld OCTA.

\section{Discussion}

\subsection{Spectrally Encoded Reflectance Imaging Performance}

The DPSL was optimized for OCT resolution while neglecting SER single-pass performance. This compromise led to 


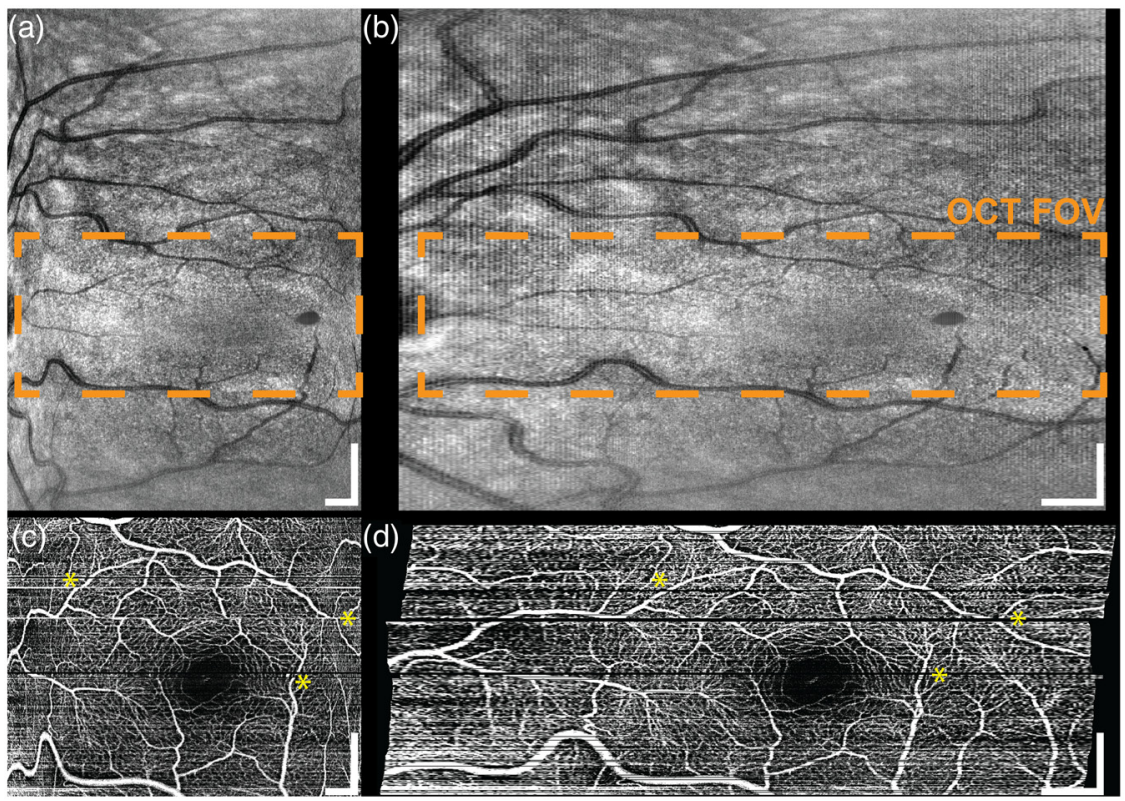

Fig. 8 SER and OCTA using variable velocity scan waveform. Four-frame averaged SER (a) before and (b) after resampling. OCTA of the fovea (c) before and (d) after motion correction and resampling. Asterisks denote motion artifacts in (c) that are corrected in (d). The lateral edges of the OCTA volumes show loss of vascular resolution as a result of undersampled angiography as compared to the densely sampled foveal region. Scale bar: $500 \mu \mathrm{m}$.

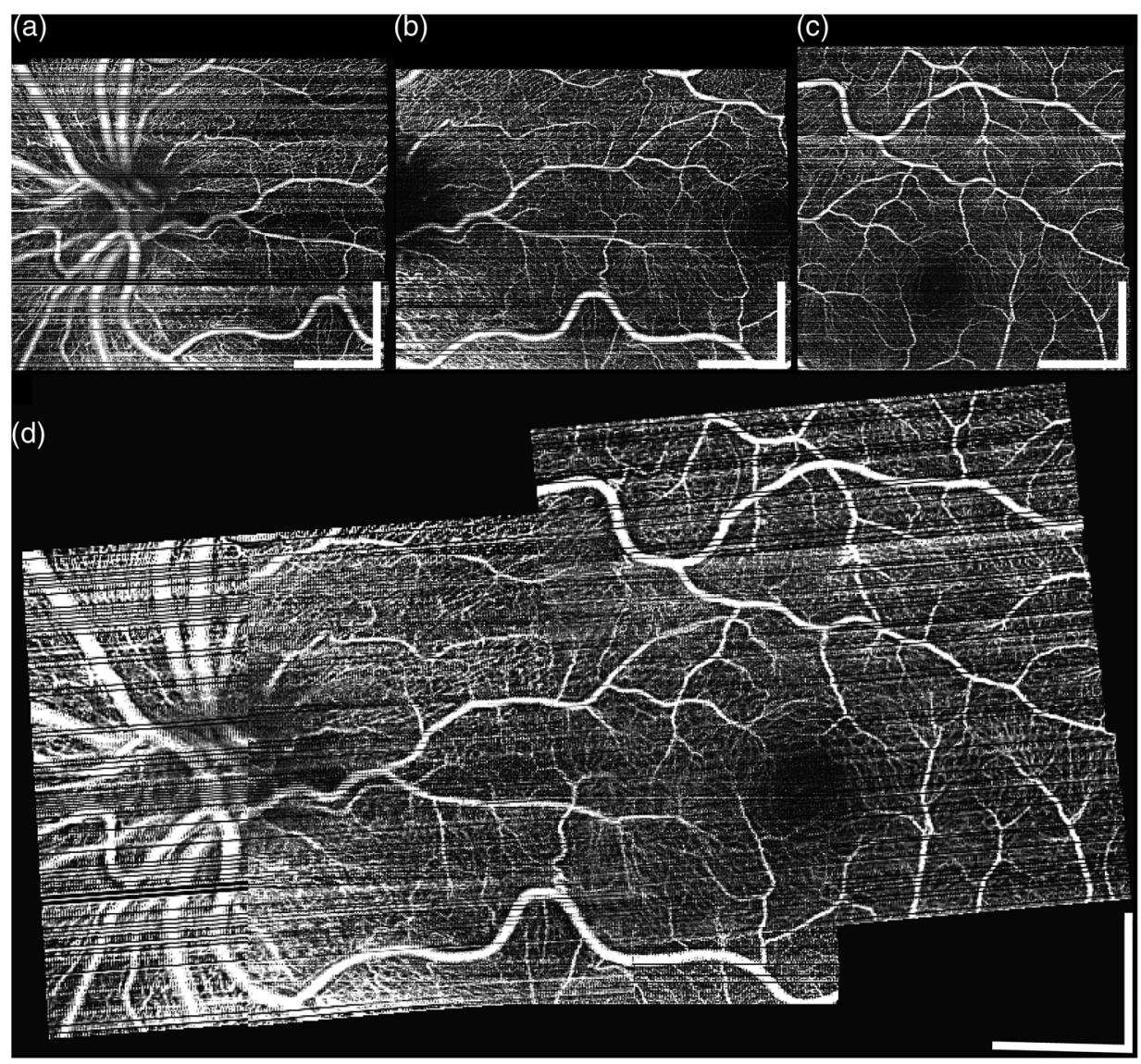

Fig. 9 Multivolume mosaic of three overlapping volumes. (a)-(c) En face projections of motion-corrected OCTA volumes from the fovea to optic nerve head and (d) corresponding mosaic. Scale bar: $1 \mathrm{~mm}$. 
significant astigmatism in the SER spots and loss of lateral resolution. However, SER performance did not significantly impact our SER-based registration and mosaicking results. While loss of SER resolution results in blurring of retinal features, any blurring of retinal vessels used for registration is assumed to be negligible relative to the scale of retinal motion artifacts.

Conversely, the presence of specular reflections resulting from lens reflections in the SER and OCT paths can negatively impact registration performance (Fig. 10 and Video 1). These artifacts reduce the contrast between retinal vessels and structure, resulting in loss of trackable fiducials. In this study, specular reflection-free regions of SER frames were isolated and used for registration (Fig. 11 and Video 2); however this required manual cropping and can potentially affect registration accuracy depending on the extent and inherent field distortions in the cropped SER region. Specular reflections can be removed from SLO or other direct-detection imaging modalities by leveraging polarization diversity between illumination and collection paths. ${ }^{49}$ However, implementing a polarization-sensitive SER system would increase system complexity and reduce optical throughput.

\subsection{Variable Velocity Waveform Utility and Mosaicking}

Noninvasive in vivo imaging of retinal microvasculature using OCTA has significantly benefited clinical ophthalmology. However, motion artifacts and trade-offs between FOV and acquisition time remain major limitations of the technology. Multivolumetric mosaicking overcomes these limitations but requires precise registration and motion correction. This is visible in the mosaic in Fig. 9(d), where excessive motion resulted in inconsistent line intensity/contrast and gaps in data that make mosaicking difficult. A nonlinear multivolumetric registration method was used in this proof-of-concept study, which necessarily deforms retinal/vascular anatomy. Nonlinear registration was used to compensate for nonlinear distortions across the FOV resulting from SECTR optics and the eye. While further work is needed to overcome these limitations, we have demonstrated the feasibility of widefield ophthalmic OCTA using a handheld probe.

In this work, we also developed a method for widefield SER and OCTA imaging using a variable velocity scan waveform. Widefield SER is necessary for aiming, localizing ROIs, and accurate motion correction, and critically sampled OCTA volumes are necessary to achieve high vascular resolution and contrast. The ability to perform OCTA imaging over arbitrarily small FOVs without affecting registration and mosaicking in postprocessing allows us to reduce total acquisition time, which is particularly advantageous when imaging patients who are unable to fixate for extended periods.

\subsection{Advantages of Spectrally Encoded Coherence Tomography and Reflectometry Imaging}

Groups have recently described handheld OCT probes and demonstrated OCTA imaging in awake and sedated infants. ${ }^{41,42}$ However, we believe the integrated motion tracking and realtime retinal aiming capabilities of SECTR provide significant advantages for point-of-care ophthalmic diagnostic imaging over these previous technologies. Here, we demonstrated an efficient motion-correction method in single OCT volumes and proof-of-concept multivolumetric mosaicking of OCTA projections. Pupil vignetting can be clearly identified on realtime SER previews to allow the system to be recentered onto the pupil, which improves the quality of the resulting OCT/ OCTA dataset. Motion correction and mosaicking also obviates the need for repeat volume acquisitions, which reduces total imaging time and would be critical for clinical translation of this technology for imaging in pediatric patients.

\section{Conclusion}

We have demonstrated a handheld SECTR imaging probe that enables volumetric OCT imaging of retinal structure and vascular perfusion in supine patients. SECTR overcomes critical barriers in image aiming, alignment, motion correction, and mosaicking and is uniquely suited for ophthalmic diagnostics

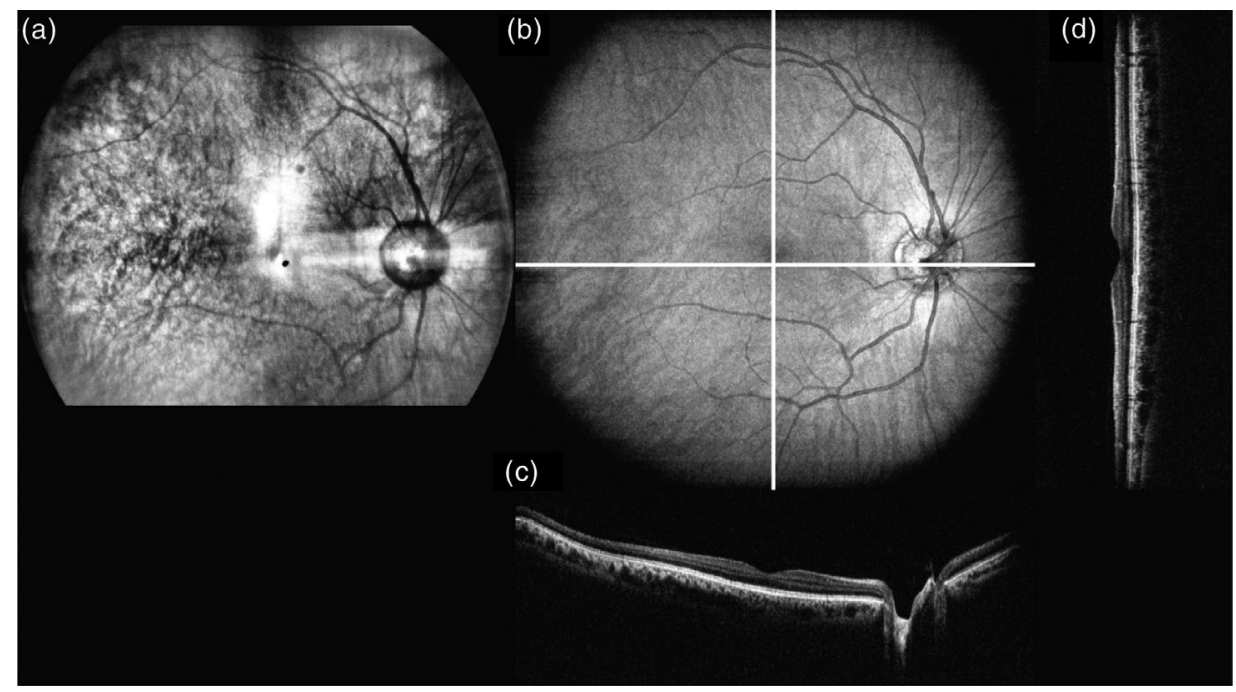

Fig. 10 Widefield in vivo human retinal imaging using handheld SECTR. (a) Raw SER frames, (b) OCT projection, and (c) fast- and (d) slow-axis OCT cross sections. The volume was sampled with $2560 \times$ $1500 \times 1500$ pixel (spectral $\times$ lines $\times$ frames) in $6.125 \mathrm{~s}$ with a frame-rate of 245 frames-per-second. SER frames show backcoupled reflection artifacts from the SECTR optics and cornea (central circular reflection) [Video 1, mp4, 8 KB (URL: https://doi.org/10.1117/1.NPh.6.4.041102.1)]. 


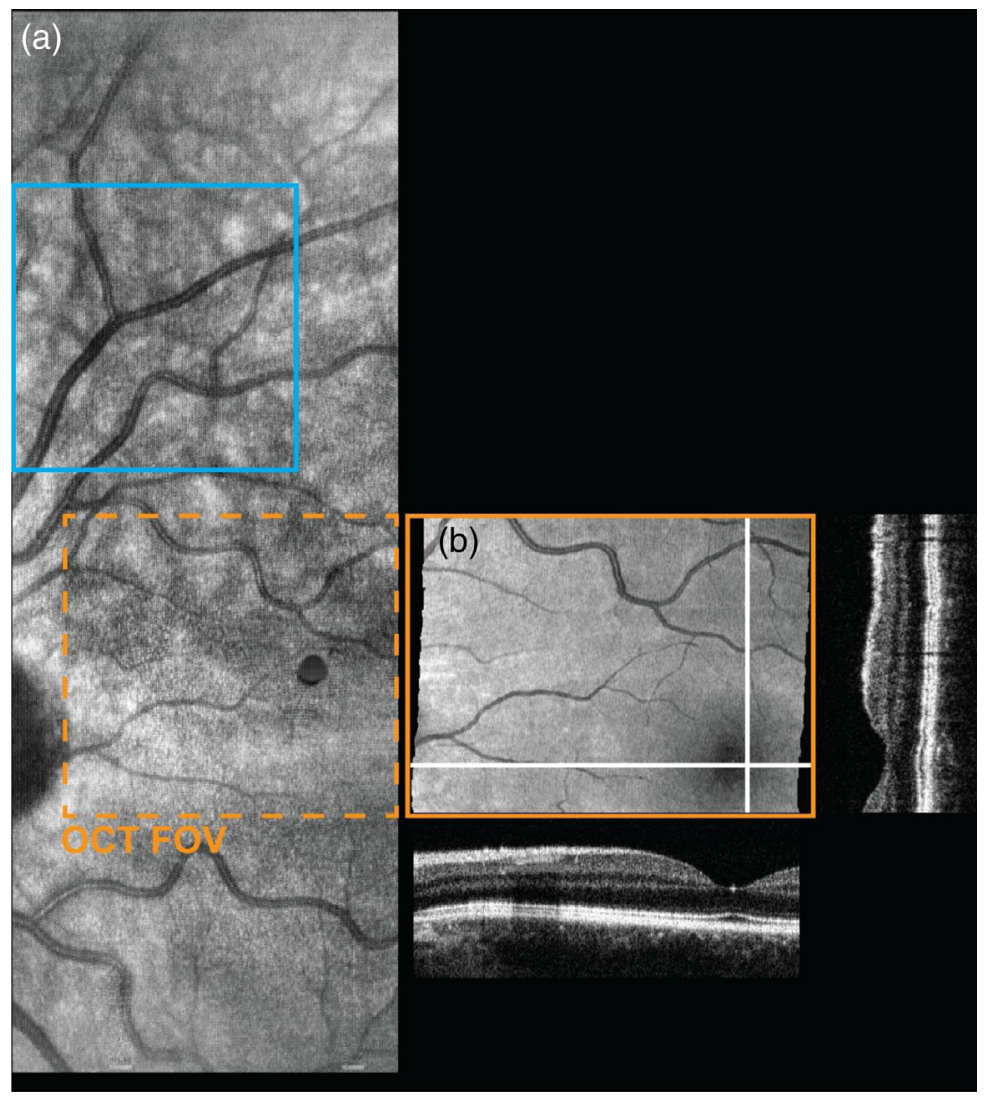

Fig. 11 In vivo human retinal OCTA imaging using handheld SECTR. Averaged SER frames (a) showing the OCT FOV (orange box) and the region used for motion-correction (blue box). The larger SER FOV enables identifying regions away from reflection artifacts that include high-contrast fiducials to optimize registration. These regions do not have to overlap with the corresponding OCT FOV, which is particularly advantageous for OCTA imaging of the central fovea, which has a limited number of vascular features. OCT average intensity projection and fast- and slow-axis cross sections (b) are shown for a volume sampled with $2560 \times 500 \times 400$ pixel (spectral $\times$ lines $\times$ frames) in $3.2 \mathrm{~s}$ [Video 2, mp4, $11 \mathrm{~KB}$ (URL: https://doi.org/10.1117/1.NPh.6.4.041102.2)].

in patients who are unable to fixate. Development of a handheld probe further extends the functionality of SECTR for supine imaging of bedridden and pediatric patients.

\section{Disclosures}

The authors have no relevant financial interests in this article and no potential conflicts of interest to disclose.

\section{Acknowledgments}

This research was supported by Vanderbilt University, the Vanderbilt Institute for Surgery and Engineering (VISE), and US National Institutes of Health Grant No. R01-EY028133. The content is solely the responsibility of the authors and does not necessarily represent the official views of the National Institutes of Health.

\section{References}

1. D. Huang et al., "Optical coherence tomography," Science 254(5035), 1178-1181 (1991).

2. C. Gerth et al., "High-resolution retinal imaging in young children using a handheld scanner and Fourier-domain optical coherence tomography," J. Am. Assoc. Pediatr. Ophthalmol. Strabismus 13(1), 72-74 (2009).
3. W. Jung et al., "Handheld optical coherence tomography scanner for primary care diagnostics," IEEE Trans. Biomed. Eng. 58(3), 741-744 (2011).

4. J. P. Campbell et al., "Handheld optical coherence tomography angiography and ultra-wide-field optical coherence tomography in retinopathy of prematurity," JAMA Ophthalmol. 135(9), 977-981 (2017).

5. C. D. Lu et al., "Handheld ultrahigh speed swept source optical coherence tomography instrument using a MEMS scanning mirror," Biomed. Opt. Express 5(1), 293-311 (2014).

6. F. LaRocca et al., "Handheld simultaneous scanning laser ophthalmoscopy and optical coherence tomography system," Biomed. Opt. Express 4(11), 2307-2321 (2013).

7. V. P. Laurutis et al., "The vestibulo-ocular reflex during human saccadic eye movements," J. Physiol. 373, 209-233 (1986).

8. R. A. Abrams et al., "Speed and accuracy of saccadic eye movements: characteristics of impulse variability in the oculomotor system," J. Exp. Psychol. Hum. Percept. Perform. 15(3), 529-543 (1989).

9. S. Martinez-Conde et al., "The role of fixational eye movements in visual perception," Nat. Rev. Neurosci. 5(3), 229-240 (2004).

10. S. H. Chavala et al., "Insights into advanced retinopathy of prematurity using handheld spectral domain optical coherence tomography imaging," Ophthalmology 116(12), 2448-2456 (2009).

11. R. J. Elble, "Central mechanisms of tremor," J. Clin. Neurophysiol. 13(2), 133-144 (1996).

12. A. Zhang et al., "Methods and algorithms for optical coherence tomography-based angiography: a review and comparison," J. Biomed. Opt. 20(10), 100901 (2015).

13. T. Huo et al., "Ultrahigh-speed optical coherence tomography utilizing all-optical 40 MHz swept-source," J. Biomed. Opt. 20(3), 030503 (2015). 
14. W. Choi et al., "Ultrahigh-speed, swept-source optical coherence tomography angiography in nonexudative age-related macular degeneration with geographic atrophy," Ophthalmology 122(12), 2532-2544 (2015).

15. T. Klein et al., "High-speed OCT light sources and systems [Invited]," Biomed. Opt. Express 8(2), 828-859 (2017).

16. M. F. Kraus et al., "Motion correction in optical coherence tomography volumes on a per A-scan basis using orthogonal scan patterns," Biomed. Opt. Express 3(6), 1182-1199 (2012).

17. P. Zang et al., "Automated motion correction using parallel-strip registration for wide-field en face OCT angiogram," Biomed. Opt. Express 7(7), 2823-2836 (2016).

18. Y. Chen et al., "Three-dimensional eye motion correction by Lissajous scan optical coherence tomography," Biomed. Opt. Express 8(3), 1783-1802 (2017).

19. Y. Chen et al., "Eye-motion-corrected optical coherence tomography angiography using Lissajous scanning," Biomed. Opt. Express 9(3), 1111-1129 (2018).

20. B. Braaf et al., "Real-time eye motion correction in phase-resolved OCT angiography with tracking SLO," Biomed. Opt. Express 4(1), 51-65 (2013).

21. M. Pircher et al., "Simultaneous SLO/OCT imaging of the human retina with axial eye motion correction," Opt. Express 15(25), 16922-16932 (2007).

22. C. K. Sheehy et al., "High-speed, image-based eye tracking with a scanning laser ophthalmoscope," Biomed. Opt. Express 3(10), 2611-2622 (2012).

23. R. Huber et al., "Fourier domain mode locking at $1050 \mathrm{~nm}$ for ultrahigh-speed optical coherence tomography of the human retina at 236, 000 axial scans per second," Opt. Lett. 32(14), 2049-2051 (2007).

24. M. Choma et al., "Sensitivity advantage of swept source and Fourier domain optical coherence tomography," Opt. Express 11(18), 2183-2189 (2003).

25. P. Zhang et al., "In vivo wide-field multispectral scanning laser ophthalmoscopy-optical coherence tomography mouse retinal imager: longitudinal imaging of ganglion cells, microglia, and Müller glia, and mapping of the mouse retinal and choroidal vasculature," J. Biomed. Opt. 20(12), 126005 (2015).

26. H.-M. Helb et al., "Clinical evaluation of simultaneous confocal scanning laser ophthalmoscopy imaging combined with high-resolution, spectral-domain optical coherence tomography," Acta Ophthalmol. 88(8), 842-849 (2010).

27. E. Moult et al., "Ultrahigh-speed swept-source OCT angiography in exudative AMD," Ophthalmic Surg. Lasers Imaging Retina 45(6), 496-505 (2014)

28. D. Nankivil et al., "Handheld, rapidly switchable, anterior/posterior segment swept source optical coherence tomography probe," Biomed. Opt. Express 6(11), 4516-4528 (2015).

29. Y. K. Tao et al., "Interlaced spectrally encoded confocal scanning laser ophthalmoscopy and spectral domain optical coherence tomography," Biomed. Opt. Express 1(2), 431-440 (2010).

30. J. D. Malone et al., "Simultaneous multimodal ophthalmic imaging using swept-source spectrally encoded scanning laser ophthalmoscopy and optical coherence tomography," Biomed. Opt. Express 8(1), 193-206 (2017).

31. M. T. El-Haddad et al., "Spectrally encoded coherence tomography and reflectometry: simultaneous en face and cross-sectional imaging at 2 gigapixels per second," J. Biophotonics 11(4), e201700268 (2018).

32. M. M. Joshi et al., "Optical coherence tomography findings in stage $4 \mathrm{~A}$ retinopathy of prematurity," Ophthalmology 113(4), 657-660 (2006).

33. C. K. Patel, "Optical coherence tomography in the management of acute retinopathy of prematurity," Am. J. Ophthalmol. 141(3), 582-584 (2006).

34. A. Vinekar et al., "Understanding clinically undetected macular changes in early retinopathy of prematurity on spectral domain optical coherence tomography," Invest. Opthalmol. Vis. Sci. 52(8), 5183-5188 (2011).

35. A. Mallipatna et al., "The use of handheld spectral domain optical coherence tomography in pediatric ophthalmology practice: our experience of 975 infants and children," Indian J. Ophthalmol. 63(7), 586-593 (2015).

36. A. Y. Tong et al., "Evaluation of optic nerve development in preterm and term infants using handheld spectral-domain optical coherence tomography," Ophthalmology 121(9), 1818-1826 (2014).
37. M. Satue et al., "Use of Fourier-domain OCT to detect retinal nerve fiber layer degeneration in Parkinson's disease patients," Eye 27(4), 507-514 (2013).

38. E. Y. J. Ng et al., "Fundus fluorescein angiography in the screening for and management of retinopathy of prematurity," J. Pediatr. Ophthalmol. Strabismus 43(2), 85-90 (2006).

39. M. T. Cabrera et al., "Exudative retinal detachment documented by handheld spectral domain optical coherence tomography after retinal laser photocoagulation for retinopathy of prematurity," Retina Cases Brief Rep. 1 (2018).

40. A. C. Lee et al., "Macular features from spectral-domain optical coherence tomography as an adjunct to indirect ophthalmoscopy in retinopathy of prematurity," Retina 31(8), 1470-1482 (2011).

41. C. Viehland et al., "Ergonomic handheld OCT angiography probe optimized for pediatric and supine imaging," Biomed. Opt. Express 10(5), 2623-2638 (2019).

42. S. Song et al., "Development of a clinical prototype of a miniature hand-held optical coherence tomography probe for prematurity and pediatric ophthalmic imaging," Biomed. Opt. Express 10(5), 2383-2398 (2019).

43. J. Polans et al., "Wide-field optical model of the human eye with asymmetrically tilted and decentered lens that reproduces measured ocular aberrations," Optica 2(2), 124-134 (2015).

44. J. Polans et al., "Asymmetric wide-field optical model of the human eye with tilted and decentered crystalline lens that reproduces experimentally measured aberrations: errata," Optica 5(11), 1461 (2018).

45. M. Guizar-Sicairos et al., "Efficient subpixel image registration algorithms," Opt. Lett. 33(2), 156-158 (2008).

46. N. Le et al., "Robust principal component analysis in optical microangiography," Quant. Imaging Med. Surg. 7(6), 654-667 (2017).

47. C. Demene et al., "Spatiotemporal clutter filtering of ultrafast ultrasound data highly increases Doppler and ultrasound sensitivity," IEEE Trans. Med. Imaging 34(11), 2271-2285 (2015).

48. A. F. Frangi et al., Multiscale Vessel Enhancement Filtering, Springer, Berlin, Heidelberg, pp. 130-137 (1998).

49. T. DuBose et al., "Handheld adaptive optics scanning laser ophthalmoscope," Optica 5(9), 1027-1036 (2018).

Joseph D. Malone received his BS degree in biomedical engineering from the University of Rochester, New York, in 2017, and is currently a PhD student at Vanderbilt University, Tennessee. He has a background in optical system design and biomedical engineering, and his doctoral research focuses on the development of ophthalmic imaging technologies to aid clinical decision-making and surgical guidance.

Mohamed T. El-Haddad received his BS degree in computer and systems engineering from Ain Shams University, Egypt, and his $\mathrm{PhD}$ in biomedical engineering from Vanderbilt University. His research focuses on the development of optical coherence tomography technologies for imaging of ophthalmic surgical dynamics.

Suhaas S. Yerramreddy is completing his BS degree in electrical engineering and computer science at Vanderbilt University. His independent research focuses on image registration and mosaicking under the mentorship of Dr. Ipek Oguz.

Ipek Oguz is an assistant professor at the Department of Electrical Engineering and Computer Science at Vanderbilt University. She received her $\mathrm{PhD}$ in computer science from the University of North Carolina at Chapel Hill. Prior to joining Vanderbilt, she spent time at the University of Pennsylvania and the University of lowa. Her research focuses on the development of methodology for quantitative medical image analysis.

Yuankai K. Tao is an assistant professor of biomedical engineering and ophthalmology and visual sciences in Vanderbilt University. His research group is affiliated with the Vanderbilt Institute for Surgery and Engineering (VISE), Vanderbilt Biophotonics Center (VBC), Vanderbilt University Institute for Imaging Science (VUIIS), and Vanderbilt Eye Institute (VEI). His research focuses on the development of optical imaging technologies with applications in ophthalmology and surgical guidance. 